\title{
Avaliação da média anual continental da concentração de material particulado
}

O material particulado (MP) é um poluente emitido na atmosfera por meio de atividades naturais e antrópicas. O MP é um dos responsáveis pela contaminação ambiental com desfechos em saúde humana, especialmente, problemas pulmonares pois, devido ao seu tamanho, atinge facilmente o trato respiratório. Nesse sentido, a Organização Mundial da Saúde (OMS) estabelece padrões mundiais relacionados aos limites de concentrações de material particulado na atmosfera. E o Grupo Banco Mundial fornece dados de renda por meio da análise de variáveis relacionadas à economia de um país, elaborando relatórios anuais para acompanhamento de seu desenvolvimento. O objetivo deste estudo foi avaliar as médias anuais continentais de concentração de MP2,5 e MP10, em 2013 e 2014, levando em consideração a Diretriz de Qualidade do Ar da Organização Mundial da Saúde e a relação da concentração do poluente com a renda de cada continente. Os resultados mostraram que a África e a Ásia têm as maiores concentrações de MP. Além disso, a maior parte das cidades estudadas excedeu o limite recomendado pela OMS e foi observado que quanto menor a renda de um continente, maior a concentração de MP, indicando que a população em países/continentes pobres está mais exposta à má qualidade do ar.

Palavras-chave: Material particulado; Qualidade do ar; Renda.

\section{Annual continental average evaluation of the particulate matter concentration}

\begin{abstract}
Particulate matter (PM) is a pollutant released in atmosphere through natural and anthropogenic activities. The PM is one of the responsible for environmental contamination with human health outcomes, especially, respiratory problems; due to the small size they can easily reach the respiratory tract. Therefore, the World Health Organization (WHO) sets global standards related to the limits of PM concentration in the atmosphere. The World Bank Group provides income data based on analyzed variables related to the country economy, preparing annual reports to monitor its development. The aim of this study was to evaluate the continental annual mean concentration of PM2,5 and PM10 in 2013 and 2014, considering the World Health Organization Air Quality Guideline and the relation between the PM concentration and the income for each continent. The results showed that Africa and Asia have higher concentration of PM. In addition, the majority of the studied cities exceeded the WHO recommended limits for PM. Moreover, it was observed that the lower is the income over the continents the higher is the PM concentration, meaning that the population in the poor countries/continents are more exposed to bad air quality.
\end{abstract}

Keywords: Particulate matter; Air quality; Income.

Topic: Desenvolvimento, Sustentabilidade e Meio Ambiente

Reviewed anonymously in the process of blind peer.

Lorrany Marins Mota (it)

Universidade Federal de Uberlândia, Brasil http://lattes.cnpq.br/1446323486851627 http://orcid.org/0000-0003-3165-013X

lorranymarins96@gmail.com

Érica Prado Domingues (iD

Universidade Federal de Uberlândia, Brasil

http://lattes.cnpq.br/5901026426732399

http://orcid.org/0000-0002-7081-9543

ericadomingues9@gmail.com

Arthur Celestino Soares

Universidade Federal de Uberlândia, Brasil http://lattes.cnpq.br/8016090842989127 http://orcid.org/0000-0002-0736-2042 arthurcelestinosoares@gmail.com
Received: 04/02/2021 Approved: 25/02/2021
Samara Carbone (iD

Universidade Federal de Uberlândia, Brasil http://lattes.cnpq.br/5664995843058837 http://orcid.org/0000-0002-3397-1183 samara.carbone@gmail.com
Referencing this:

MOTA, L. M.; DOMINGUES, É, P.; SOARES, A. C.; CARBONE, S. Avaliação da média anual continental da concentração de material particulado. Revista Ibero Americana de Ciências Ambientais, v.12, n.2, p.530-538, 2021. DOI: http://doi.org/10.6008/CBPC2179$\underline{6858.2021 .002 .0045}$

DOI: 10.6008/CBPC2179-6858.2021.002.0045 


\section{INTRODUÇÃO}

A presença de poluentes na atmosfera compromete a qualidade do ar e gera riscos para o ecossistema e para a saúde humana (CIARELLI et al., 2016), sendo as atividades humanas, tais como queima de biomassa, detritos de solo, emissões industriais e tráfego veicular algumas das responsáveis por emitir contaminantes na atmosfera (BAIRD et al., 2011; RODRIGUES et al., 2017).

O material particulado (MP) consiste numa mistura complexa de partículas sólidas e/ou líquidas e é um dos poluentes atmosféricos responsáveis pelo tal comprometimento do ar (SEINFELD et al., 2016; VARDAR et al., 2014; KARAGULIAN et al., 2015; BARI et al., 2018) e pode ser dividido de acordo com o diâmetro de suas partículas. Recebe a denominação de $\mathrm{MP}_{10}$ aquelas partículas com diâmetro inferior a 10 $\mu \mathrm{m}$ e de $\mathrm{MP}_{2,5}$ as partículas finas menores do que 2,5 $\mu \mathrm{m}$. Além disso, o MP é formado por partículas inaláveis que se encontram suspensas na atmosfera e dentre os diversos compostos que podem ser encontrados em sua composição, pode-se citar sulfato, nitrato, amônio, cloreto, black carbon (parte da fração carbonácea do MP emitida durante processos de combustão incompleta), partículas minerais e água (SEINFELD et al., 2006).

Diversos estudos apresentam resultados dos efeitos da poluição do ar na saúde da população (BARI et al., 2018; CIARELLI et al., 2016; FREITAS et al., 2016; KARAGULIAN et al., 2015; REIS et al., 2017; RIZKY et al., 2016; RODRIGUES et al., 2017; VARDAR et al., 2014), sendo também relacionados às cidades com elevado número de pessoas (GUZMÁN et al., 2017). Devido ao seu diâmetro micrométrico, o MP pode rápida e facilmente seja inalado (IPCC, 2014) e, segundo Braga et al. (2001), a partícula pode adsorver alguns gases em sua superfície, podendo também ser transportados até o sistema respiratório. E dentre as duas divisões citadas anteriormente, o $\mathrm{MP}_{2,5}$ é aquele que apresenta maior risco à saúde humana devido ao seu diâmetro menor, podendo atingir com mais facilidade regiões mais profundas do trato respiratório (RODRIGUES et al., 2017).

A exposição aos poluentes atmosféricos aponta para aumento no número de internações devido a agravos no sistema respiratório e cardiovascular com desfechos em lesões pulmonares e infarto, adversidades durante a gravidez, como prematuridade, restrição do crescimento e baixo peso ao nascer, e mortalidade quando existem complicações (RODRIGUES et al., 2017; FREITAS et al., 2016; REIS et al., 2017; RIZKY et al., 2016). Os efeitos na saúde variam conforme a composição química, período de exposição e concentração dos poluentes atmosféricos (ALVES et al., 2015).

No que diz respeito à saúde ambiental, a Organização Mundial da Saúde (OMS) desenvolve estudos e estabelece valores para os padrões mundiais de qualidade ambiental, incluindo padrões de qualidade do ar com limites de concentrações de poluentes atmosféricos (WHO, 2006). Um tema relevante quando se analisa a saúde ambiental, assim como a saúde de uma determinada população, diz respeito ao nível de renda desse local. Pode se afirmar que os níveis de renda no mundo apresentam disparidades importantes e pode-se verificar também tais disparidades entre os diferentes continentes. Neste sentido, além de examinar a concentração de MP em nível mundial, torna-se relevante associar estes dados com o nível de renda das populações, componente importante do padrão de desenvolvimento dos países. 
Nesse sentido, o Grupo Banco Mundial é uma agência que fornece assistência para os países ao disponibilizar recursos financeiros para o seu desenvolvimento, sendo seu principal objetivo reduzir a pobreza e as desigualdades. O Banco Mundial une o financiamento com uma consultoria política, visando o desenvolvimento sustentável (BANCO MUNDIAL, 2015) e, anualmente, a agência elabora relatórios com o panorama do desenvolvimento mundial, incluindo dados sobre a renda de cada país.

Diante desse contexto, o presente trabalho teve por objetivo realizar um levantamento anual das médias de concentração de $\mathrm{MP}_{2,5}$ e $\mathrm{MP}_{10}$ por continente, entre 2013 e 2014, para avaliar locais com concentração maior do que o estabelecido pela Diretriz de Qualidade do Ar da Organização Mundial da Saúde e relacionar com a renda de cada continente. Assim, pode-se avançar neste tema ainda tão urgente aos países pobres e em desenvolvimento, ou seja, um processo de desenvolvimento socioeconômico que seja de fato sustentável e que em última análise apresenta-se como uma necessidade para o mundo como um todo.

\section{MATERIAIS E MÉTODOS}

\section{Base de dados}

A OMS possui uma base de dados com informações de concentrações anuais de $\mathrm{MP}_{2,5} \mathrm{e} \mathrm{MP}_{10}$ de inúmeras cidades ao redor do mundo, havendo estimativas dos valores caso não houvesse registro das concentrações ${ }^{1}$. Segundo a OMS, cada cidade recebe uma classificação conforme sua renda e dados de concentração de MP nas cidades, sendo estes dados enviados de várias estações de monitoramento da qualidade do ar espalhadas pelo planeta e disponibilizados para o uso público.

As informações sobre a renda foram retiradas da base de dados do Grupo Banco Mundial², baseado em sua própria metodologia que leva em consideração algumas variáveis como população, produto interno bruto, inflação e moeda local, sendo as opções de classificações de renda: baixa, média-baixa, média-alta e alta (WHO, 2016).

No presente estudo, foi feita uma análise das médias anuais continentais da poluição do ar, bem como a relação com os aspectos sociais através da renda. Devido a quantidade significativa de informações disponíveis na plataforma da OMS para as médias anuais de concentração de $\mathrm{MP}_{2,5}$ e $\mathrm{MP}_{10}$, neste trabalho serão utilizados dados de 2.610 cidades distribuídas nos continentes África, América, Ásia, Europa e Oceania, entre os anos de 2013 e 2014.

As cidades foram separadas e trabalhadas por continente (Tabela 1 ), por ser um modelo mais simples e de fácil entendimento, diferentemente da base de dados da OMS que divide o mundo por regiões, sendo elas: Europa, Pacífico Ocidental, África, América, Mediterrâneo Oriental e Sudeste Asiático.

Tabela 1: Renda, países e número de cidades por continente.

\begin{tabular}{lllr}
\hline Continentes & Renda & Países & $\begin{array}{c}\text { No } \\
\text { cidades }\end{array}$ \\
\hline \multirow{2}{*}{ África } & Baixa & Nigéria, Uganda & 20 \\
\hline
\end{tabular}

${ }^{1}$ http://www.who.int/gho/phe/outdoor air pollution/exposure/en/

2 https://datahelpdesk.worldbank.org/knowledgebase/articles/378832-what-is-the-world-bank-atlas-method 


\begin{tabular}{|c|c|c|c|}
\hline & \multicolumn{2}{|l|}{ baixa } & \\
\hline & $\begin{array}{l}\text { Média- } \\
\text { alta }\end{array}$ & África do Sul & \\
\hline \multirow{3}{*}{ América } & $\begin{array}{l}\text { Média- } \\
\text { baixa }\end{array}$ & Bolívia, El Salvador, Guatemala, Honduras & \multirow{3}{*}{599} \\
\hline & $\begin{array}{l}\text { Média- } \\
\text { alta }\end{array}$ & Brasil, Colômbia, Costa Rica, Equador, México, Panamá, Peru & \\
\hline & Alta & Canadá, Chile, Estados Unidos da América, Uruguai & \\
\hline \multirow{4}{*}{ Ásia } & Baixa & Nepal & \multirow{4}{*}{328} \\
\hline & $\begin{array}{l}\text { Média- } \\
\text { baixa }\end{array}$ & Bangladesh, Índia, Indonésia, Myanmar, Filipinas & \\
\hline & $\begin{array}{l}\text { Média- } \\
\text { alta }\end{array}$ & Singapura, China, Irã, Líbano, Malásia, Tailândia & \\
\hline & Alta & Kuwait, República da Coréia, Arábia Saudita, Emirados Árabes Unidos & \\
\hline \multirow{3}{*}{ Europa } & $\begin{array}{l}\text { Média- } \\
\text { baixa }\end{array}$ & Geórgia & \multirow{3}{*}{1626} \\
\hline & $\begin{array}{l}\text { Média- } \\
\text { alta }\end{array}$ & Albânia, Bulgária, Sérvia, Montenegro, Romênia, República da Macedônia & \\
\hline & Alta & $\begin{array}{l}\text { Andorra, Áustria, Bélgica, Croácia, Chipre, República Tcheca, Dinamarca, Estônia, Finlândia, } \\
\text { França, Alemanha, Grécia, Hungria, Islândia, Irlanda, Israel, Itália, Letônia, Lituânia, } \\
\text { Luxemburgo, Malta, Mônaco, Países Baixos, Noruega, Polônia, Portugal, Eslováquia, } \\
\text { Eslovênia, Espanha, Suécia, Suíça, Reino Unido }\end{array}$ & \\
\hline Oceania & Alta & Austrália & 37 \\
\hline
\end{tabular}

Fonte: WHO (2016).

\section{Análise estatística}

Inicialmente, as cidades do estudo foram agrupadas por continentes e foi analisado o valor mínimo e máximo de MP dos continentes, para verificar se os mesmos estão respeitando os valores médios anuais de concentração estabelecidos pela OMS, que são de 10 e $20 \mu \mathrm{g} \mathrm{m}^{-3}$, respectivamente, para $\mathrm{MP}_{2,5}$ e $\mathrm{MP}_{10}$ (WHO, 2006).

Para analisar a proporção de cidades que estão respeitando os padrões de concentração mundial ditados pela OMS, calculou-se a porcentagem para cada continente, levando em consideração o número de cidades relativas a cada continente nos anos de 2013 e 2014. E por fim, foi calculado o valor de tendência para cada continente, descrito pelo coeficiente de determinação $\left(R^{2}\right)$, que varia entre 0 e 1 , sendo que quanto maior for o valor do coeficiente, mais os dados são explicados pela tendência do modelo e, logo, se correlacionam.

\section{RESULTADOS E DISCUSSÃO}

As concentrações médias de $\mathrm{MP}_{10}$ e $\mathrm{MP}_{2,5}$ variaram em relação a localidade, sendo os continentes África e Ásia aqueles que possuíram as mais elevadas concentrações desses poluentes (Tabela 2). É possível observar que em todos continentes a concentração de $\mathrm{MP}_{10}$ foi mais elevada do que a de $\mathrm{MP}_{2,5}$, conforme esperado. Isto acontece porque pela definição na obtenção do $\mathrm{MP}_{10}$ é levada em consideração partículas de diâmetros maiores, o que implica em um aumento da concentração de massa. A África, em particular, apresentou maior média anual de concentração de $\mathrm{MP}_{10}\left(93 \mu \mathrm{g} \mathrm{m}^{-3}\right)$ e elevada variação, com mínimo de 24 $\mu \mathrm{g} \mathrm{m}^{-3}$, na cidade de Middleburg, na África do Sul, e máximo de $423 \mu \mathrm{g} \mathrm{m}^{-3}$, devido à grande concentração de poluentes na cidade de Kaduna, na Nigéria. Além disso, a cidade de cidade de Middleburg, na África do Sul, apresentou a menor concentração de $\mathrm{MP}_{2,5}\left(13 \mu \mathrm{g} \mathrm{m}^{-3}\right)$ e a cidade de Kampala, na Uganda, a maior (104 $\mu \mathrm{g}$ 
$\left.\mathrm{m}^{-3}\right)$.

Tabela 2: Mínimos, médias, máximos de concentração de $\mathrm{MP}_{10}$ e $\mathrm{MP}_{2,5}$ e porcentagem de cidades que ultrapassam o limite por continentes

\begin{tabular}{|c|c|c|c|c|c|c|c|c|c|}
\hline & $\mathrm{MP}_{10}$ & & & & & $\mathrm{MP}_{2,5}$ & & & \\
\hline Continentes & $\begin{array}{l}\text { Mínimo } \\
\left(\mu \mathrm{g} \mathrm{m}^{-3}\right)\end{array}$ & $\begin{array}{l}\text { Média } \\
\left(\mu \mathrm{g} \mathrm{m}^{-3}\right)\end{array}$ & $\begin{array}{l}\text { Máximo } \\
\left(\mu \mathrm{g} \mathrm{m}^{-3}\right)\end{array}$ & $\begin{array}{l}\text { Cidades } \\
\text { ultrapassam } \\
\text { limite (\%) }\end{array}$ & $\begin{array}{r}\text { que } \\
0\end{array}$ & $\begin{array}{l}\text { Mínimo } \\
\left(\mu \mathrm{g} \mathrm{m}^{-3}\right)\end{array}$ & $\begin{array}{l}\text { Média } \\
\left(\mu \mathrm{g} \mathrm{m}^{-3}\right)\end{array}$ & $\begin{array}{l}\text { Máximo } \\
\left(\mu \mathrm{g} \mathrm{m}^{-3}\right)\end{array}$ & $\begin{array}{lr}\text { Cidades } & \text { que } \\
\text { ultrapassam } & \text { o } \\
\text { limite (\%) } & \end{array}$ \\
\hline África & 24 & 93 & 423 & $100 \%$ & & 13 & 39 & 104 & $100 \%$ \\
\hline América & 3 & 19 & 95 & $19,8 \%$ & & 2 & 10 & 64 & $22,0 \%$ \\
\hline Ásia & 16 & 85 & 368 & $99,1 \%$ & & 10 & 49 & 156 & $99,7 \%$ \\
\hline Europa & 4 & 24 & 140 & $58,1 \%$ & & 2 & 15 & 81 & $77,2 \%$ \\
\hline Oceania & 13 & 18 & 22 & $16,2 \%$ & & 5 & 7 & 10 & $0 \%$ \\
\hline
\end{tabular}

No continente Americano, os valores mínimo e máximo de concentração de $\mathrm{MP}_{10}$ foram as cidades de Sinclair, nos Estados Unidos $\left(3 \mu \mathrm{g} \mathrm{m}^{-3}\right)$, e Santa Gertrudes, no Brasil $\left(95 \mu \mathrm{g} \mathrm{m}^{-3}\right)$, respectivamente; e para as concentrações de $\mathrm{MP}_{2,5}$, foram Sinclair, nos Estados Unidos $\left(2 \mu \mathrm{g} \mathrm{m}^{-3}\right)$, e Coihaque, no Chile $\left(64 \mu \mathrm{g} \mathrm{m}^{-3}\right)$. Já no continente Asiático, as concentrações mínimas e máximas para $\mathrm{MP}_{10}$ se referem às cidades de Taitung, na China $\left(16 \mu \mathrm{g} \mathrm{m}^{-3}\right)$, e Riyadh, na Arábia Saudita $\left(368 \mu \mathrm{g} \mathrm{m}^{-3}\right)$, e para $\mathrm{MP}_{2,5}$, Tanah Rata, na Malásia $\left(10 \mu \mathrm{g} \mathrm{m}^{-3}\right)$, e Riyadh, na Arábia Saudita $\left(156 \mu \mathrm{g} \mathrm{m}^{-3}\right)$, respectivamente.

Na Europa, a cidade de Muonio, na Finlândia, teve as concentrações mínimas para $\mathrm{MP}_{10}\left(4 \mu \mathrm{g} \mathrm{m}^{-3}\right)$ e para $\mathrm{MP}_{2,5}\left(2 \mu \mathrm{g} \mathrm{m}^{-3}\right)$ e a cidade de Tetovo, na Macedônia, apresentou as máximas concentrações para $\mathrm{MP}_{10}$ $\left(140 \mu \mathrm{g} \mathrm{m}^{-3}\right)$ e $\mathrm{MP}_{2,5}\left(81 \mu \mathrm{g} \mathrm{m}^{-3}\right)$. E na Oceania, os valores mínimo e máximo de concentração de $\mathrm{MP}_{10}$ foram as cidades de Central Coast, na Austrália $\left(13 \mu \mathrm{g} \mathrm{m}^{-3}\right)$, e Geraldton, na Austrália $\left(22 \mu \mathrm{g} \mathrm{m}^{-3}\right)$, respectivamente e para $\mathrm{MP}_{2,5}$ foram as cidades de Gladstone, na Austrália $\left(5 \mu \mathrm{g} \mathrm{m}^{-3}\right)$, e Geraldtond e Vale Latrobe, na Austrália $\left(10 \mu \mathrm{g} \mathrm{m}^{-3}\right)$

Ainda segundo a Tabela 2, todas as 20 cidades do continente Africano utilizadas neste estudo apresentaram concentrações de material particulado acima do limite estabelecido pela OMS de 20 e $10 \mu \mathrm{g}$ $\mathrm{m}^{-3}$, respectivamente, para $\mathrm{MP}_{10}$ e $\mathrm{MP}_{2,5}$. Esta situação se repete na Ásia, onde 99,1\% das cidades (325 cidades) ultrapassam o limite de $\mathrm{MP}_{10}$ e 99,7\% (327 cidades) o limite para $\mathrm{MP}_{2,5}$.

No continente Europeu, 58,1\% das cidades analisadas (945 cidades) ultrapassam a concentração estabelecida pela OMS, para $\mathrm{MP}_{10}$, e 77,2\% (1.255 cidades), para $\mathrm{MP}_{2,5}$. Nos continentes América e Oceania, a situação encontrada não é tão crítica quanto nos demais continentes. Na América, 119 cidades (19,8\%) e 132 cidades (22\%) estão, respectivamente, acima do limite para $\mathrm{MP}_{10}$ e $\mathrm{MP}_{2,5}$; e na Oceania, 6 cidades ultrapassam o limite para $\mathrm{MP}_{10}(16,2 \%)$ e nenhuma das 37 cidades utilizadas no estudo têm concentração superior ao limite de $10 \mu \mathrm{g} \mathrm{m}^{-3}$ para $\mathrm{MP}_{2,5}$.

Os fatores responsáveis pelos elevados valores encontrados de concentração de MP na atmosfera são muito distintos e variam devido ao intenso fluxo veicular, principalmente, veículos pesados que utilizam diesel como combustível, a queima de carvão mineral, emissões industriais, queima de lenha para geração de energia e para preparo de alimentos (BAIRD et al., 2011). No entanto, estudos mostram que tipos de fontes emissoras de poluentes atmosféricos, tais como o MP, são bastante distintas de uma localidade para outra e estão fortemente relacionados à renda, e, portanto, a grau de desenvolvimento daquela região ou 
país.

O relatório da OMS (WHO, 2018) mostra que fontes oriundas do ambiente doméstico, tais como queima de biomassa (lenha e carvão) para aquecimento e preparo de alimentos, estão associadas à morte de 3.8 milhões de pessoas em 2016. Sendo que mais de 90\% destas mortes ocorreram em países de renda baixa e média, principalmente países da Ásia e África. Por exemplo, na China, aproximadamente $20 \%$ das mortes por má qualidade do ar estão associadas ao uso de carvão mineral para geração de energia nos processos industriais, um país com renda média-alta conforme a classificação do Banco Mundial (2015). Já na Índia, um país de renda média-baixa, $40 \%$ das mortes associadas à má qualidade do ar são de queima de biomassa residencial para produção de energia e preparo de alimentos (HEI, 2019).

A queima de lenha, carvão e esterco de animais, nas ruas e dentro de casa, como forma de produção de energia para preparo de alimentos é uma técnica bastante comum nos países subdesenvolvidos e em desenvolvimento (WHO, 2018). Enquanto isto, países mais desenvolvidos, tais como os Estados Unidos e Alemanha, países estes com renda alta, apresentam como principais fontes de emissão de MP indústrias e queima de combustíveis fósseis para produção de energia e transporte (EPA, 2018).

Existem casos isolados, como por exemplo, indústrias de produção de cerâmica na cidade de Santa Gertrudes, localizada no estado de São Paulo, no Brasil, um pólo cerâmico com 32 indústrias (OLIVEIRA et al., 2016) e elevadas concentrações de MP num país de média-baixa renda. No entanto, os valores nas regiões com baixa concentração de MP podem ser descritos pelo maior investimento em tecnologias limpas que são menos agressivas ao ambiente, sendo a Finlândia um exemplo de país no quesito sustentabilidade e, conforme descrito por Figueiredo (1994), o investimento tecnológico gera um desenvolvimento mais sustentável.

O investimento em tecnologias limpas é um fator importante, mas a diferença de renda e padrões de desenvolvimento entre os países mantém desigualdades em vários aspectos, dentre eles os padrões de produção e consumo vigentes nos diferentes países e continentes, para os quais a tecnologia deverá ser apropriada aos diferentes níveis de renda e desenvolvimento. O processo de desenvolvimento dos países sub ou em desenvolvimento deve ser entendido no contexto da expansão do capitalismo mercantil europeu que colonizou boa parte deles, o que criou uma dependência dos mesmos para com o sistema internacional que privilegia os países detentores de capital, tecnologia e mercados.

E para que estas desigualdades sejam amenizadas, uma oportunidade se faz presente atualmente, com a necessidade de atuar sobre a problemática ambiental em nível mundial, mas considerando-se as necessidades e especificidades dos vários países e continentes, que mude as diferenças de renda ainda existentes entre eles. Tal discussão requer uma nova concepção de desenvolvimento, onde a associação de uma concepção válida de sustentabilidade seja associada à de desenvolvimento, mas que este seja socioeconômico, ético e político-cultural com a devida justiça social reclamada no século XXI.

Ao analisar a distribuição da população global pode-se observar que o continente Asiático detém mais da metade de toda população global (59,63\%), seguida pela África (16,59\%), América (13,33\%), Europa 
$(9,89 \%)$ e Oceania $(0,56 \%)^{3}$. E Krzyzanowski et al. (2014), descreveram que as concentrações de MP tendem a aumentar com o aumento da densidade populacional de uma região.

A urbanização pode ter impactos tanto positivos quanto negativos em países em desenvolvimento; países como a China, que tiveram um alto grau de crescimento econômico em um curto espaço de tempo, acarretam numa série de mudanças ao ambiente, como por exemplo, o aumento da urbanização, que contribui com a poluição do ambiente (HAN et al., 2015).

Na Figura 1, é observada uma tendência de decréscimo da concentração de MP conforme a renda aumenta. Os valores de tendência, descritos pelo coeficiente de determinação, para a África foram de 0,9 para $\mathrm{MP}_{10}$ e 0,9 para $\mathrm{MP}_{2,5}$, para a Ásia foi de 0,2 para $\mathrm{MP}_{10}$ e 0,002 para $\mathrm{MP}_{2,5}$, para a América foi de 1,0 para $\mathrm{MP}_{10}$ e 0,9 para $\mathrm{MP}_{2,5}$, para a Europa foi de 0,9 para $\mathrm{MP}_{10}$ e 0,6 para $\mathrm{MP}_{2,5}$ e a Oceania se apresenta como exceção, tendo em vista que o continente apresentou apenas dados para renda alta, não sendo possível haver comparações com as demais rendas. Estes coeficientes demonstram que quanto mais próximo o valor for de 1, mais os dados observados são explicados pela tendência de redução da concentração do MP com o aumento da renda.

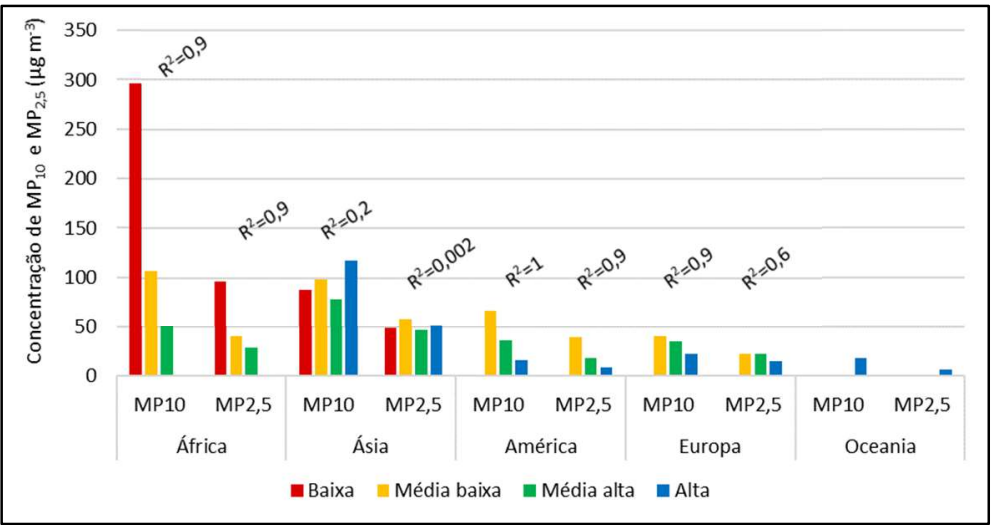

Figura 1: Concentração média de $\mathrm{MP}_{10}$ e $\mathrm{MP}_{2,5}$ emitida por continente de acordo com a renda.

Além disso, no continente Asiático, a concentração de MP não acompanhou tal tendência, pois apresentava dois blocos de países muito distintos um do outro, onde a concentração média dos países na região do Mediterrâneo Oriental ( $171 \mu \mathrm{g} \mathrm{m}^{-3}$ ) foi mais elevada do que a média na região do Pacífico Ocidental $\left(46 \mathrm{~g} \mathrm{~m} \mathrm{~m}^{-3}\right)$. Neste estudo, optou-se pelo uso da divisão por continentes geográficos por permitir resultados mais simples, ao invés da divisão por grupos de países feita pela OMS, embora esta última trate de um problema amplamente complexo, uma vez que leva em consideração a disparidade de rendas dentro do mesmo continente geográfico.

Ao comparar os valores médios de concentrações de MP dos dados continentais foi possível observar que continentes com maior desenvolvimento econômico apresentam menores concentrações dos poluentes tanto para $\mathrm{MP}_{10}$ quanto para $\mathrm{MP}_{2,5}$. Esse modelo corrobora dados descritos por Mage et al. (1996) e Krzyzanowski et al. (2014).

${ }^{3}$ www.statista.com/statistics/237584/distribution-of-the-world-population-by-continent/ 


\section{CONCLUSÕES}

As análises estatísticas permitiram identificar os continentes com concentrações de $\mathrm{MP}_{2,5} \mathrm{e} \mathrm{MP}_{10}$ acima dos limites estabelecidos pela OMS, de 10 e $20 \mathrm{\mu g} \mathrm{m}^{-3}$, respectivamente. Os continentes África e Ásia apresentam maiores concentrações de MP, a maioria das cidades do estudo ultrapassam os limites estabelecidos pela OMS e existe uma tendência de decréscimo da concentração de material particulado conforme a renda aumenta.

Os métodos para avaliação da qualidade do ar que quantificam o impacto da poluição atmosférica na saúde estão se desenvolvendo rapidamente, resultando em mais ações efetivas para conter a poluição do ar e a redução de impactos a saúde. Embora existam locais onde a emissão de poluentes do ar ultrapasse os limites legais estabelecidos com menor frequência, tais como Austrália na Oceania, vale lembrar que ainda se faz necessário o monitoramento da qualidade do ar nas cidades visando a implementação de políticas públicas que visem a melhora da qualidade de vida, pois um grande número de pessoas está submetido a níveis que qualidade do ar inferiores aos recomendados.

E com relação à renda, as análises sobre as desigualdades nesta, mas também em outras variáveis que contribuem para sua reprodução entre países e continentes, indicam a necessidade urgente de redução das desigualdades para que um nivelamento ocorra entre os mesmos e favoreçam esta redução. Para isto, faz-se necessário uma nova concepção de desenvolvimento, e de sustentabilidade na teoria e na prática, e a associação entre ambos para que a saúde ambiental e níveis de renda permitam a resolução dos problemas ambientais e de pobreza no mundo.

\section{REFERÊNCIAS}

ALVES, D. D.; OSÓRIO, D. M. M.; RODRIGUES, M. A. S.; ILLI, J. C.; BIANCHIN, L.; BENVENUTI, T.. Concentrations of PM 2.5-10 and $\mathrm{PM}_{2.5}$ and metallic elements around the Schmidt Stream area, in the Sinos River Basin, southern Brazil. Brazilian Journal of Biology, São Carlos, v.75, n.4, p.43-52, 2015. DOI: http://doi.org/10.1590/1519-6984.00113suppl

BAIRD, C.; CANN, M.. Química Ambiental. 4 ed. Porto Alegre: Bookman, 2011.

BANCO MUNDIAL. Relatório Anual de 2015 do Banco Mundial. Washington: World Bank Group, 2015.

BARI, A.; KINDZIERSKI, W. B.. Characterization of air quality and sources of fine particulate matter $\left(\mathrm{PM}_{2.5}\right)$ in the City of Calgary, Canada. Atmospheric Pollution Research, Izmir, v.9, p.534-543, 2018. DOI:

http://doi.org/10.1016/j.apr.2017.11.014

BRAGA, A.; BÖHM, G. M.; PEREIRA, L. A. A.; SALDIVA, P.. Poluição atmosférica e saúde humana. Revista USP, São Paulo, n.51, p.58-71, 2001. DOI:

http://doi.org/10.11606/issn.2316-9036.v0i51p58-71

CIARELLI, G.; AKSOYOGLU, S.; CRIPPA, C.; JIMENEZ, J-L.; NEMITZ, E.; SELLEGRI, K.; ÄIJÄLÄ, M.; CARBONE, S.; MOHR, C.; O'DOWD, C.; POULAIN, L.; BALTENSPERGER, U.; PRÉVÔT, A. S. H.. Evaluation of European air quality modelled by
CAMx including the volatility basis set scheme. Atmospheric Chemistry and Physics, Katlenburg-Lindau, v.16, p.1031310332, 2016. DOI: http://doi.org/10.5194/acp-16-10313$\underline{2016}$

EPA. Environmental Protection Agency. 2014 National Emissions Inventory, version 2: Technical Support Document. North Carolina: EPA, 2018.

FIGUEIREDO, P. J. M.. A sociedade do lixo: Os resíduos, a questão energética e a crise ambiental. Campinas: UNIMEP, 1994.

FREITAS, C. U.; LEON, A. P.; JUNGER, W.; GOUVEIA, N.. Air pollution and its impacts on health in Vitoria, Espírito Santo, Brazil. Revista de Saúde Pública, São Paulo, v.50, n.4, p.1-9, 2016. DOI: http://doi.org/10.1590/S1518$\underline{8787.2016050005909}$

GUZMÁN, A. A. E.; TZUC, O. M.; PANTÍ, I. B.; TRUJEQUE, J. R.; QUINTANA, I. V. P.; BASSAM, A.. Modelado de partículas $\mathrm{PM}_{10}$ y $\mathrm{PM}_{2.5}$ mediante redes neuronales artificiales sobre clima tropical de San Francisco de Campeche, México. Química Nova, São Paulo, v.40, n.9, p.1025-1034, 2017. DOI: http://doi.org/10.21577/0100-4042.20170115

HAN, L.; ZHOU, W.; LI, W.. Increasing impact of urban fine particles $\left(\mathrm{PM}_{2.5}\right)$ on areas surrounding Chinese cities. 
Scientific Reports, Londres, v.5, n.1, 2015. DOI: http://doi.org/10.1038/srep12467

HEl. Health Effects Institute. State of Global Air 2019. Special Report. Boston: HEI, 2019.

IPCC. Painel Intergovernamental de Mudanças Climáticas. Climate Change 2014: Mitigation of Climate Change. Cambridge, United Kingdom e New York, USA: Cambridge University Press, 2014

KARAGULIAN, F.; BELIS, C. A.; DORA, C. F. C.; PRÜSS-USTÜN, A. M.; BONJOUR, S.; ADAIR-ROHANI, H.; AMANN, M. Contributions to cities' ambient particulate matter (PM): A systematic review of local source contributions at global level. Atmospheric Environment, Oxford, v.120, p.475-483, 2015. DOI: http://doi.org/10.1016/j.atmosenv.2015.08.087

KRZYZANOWSKI, M.; APTE, J. S.; BONJOUR, S. P.; BRAUER, M.; COHER, A. J.; PRÜSS-USTÜN, A. M.. Air pollution in the mega-cities. Current Environmental Health Reports, Suiça, v.1, n.3, p.185-191, 2014. DOI:

https://doi.org/10.1007/s40572-014-0019-7

MAGE, D.; OZOLINS, G.; PETERSON, P.; WEBSTER, A.; ORTHOFER, R.; VANDEWEERD, V.; GWYNNE, M.. Urban air pollution in mega-cities of the world. Atmospheric Environment, Oxford, v.30, n.5, p.681-686, 1996. DOI: http://doi.org/10.1016/1352-2310(95)00219-7

OLIVEIRA, M.; ZANARDO, A.; CARVALHO, S. G.; ROCHA, R. R. Características mineralógicas e granulométricas das fontes de poeiras no Polo Cerâmico de Santa Gertrudes - SP. Cerâmica, São Paulo, v.62, p.198-205, 2016. DOI: http://doi.org/10.1590/0366-69132016623621973

REIS, M. M.; GUIMARÃES, M. T.; BRAGA, A. L. F.; MARTINS, L. C.; PEREIRA, L. A. A.. Air pollution and low birth weight in an industrialized city in Southeastern Brazil, 2003-2006. Revista Brasileira de Epidemiologia, São Paulo, v.20, n.2, p.189-199, 2017. DOI: http://doi.org/10.1590/1980-5497201700020001

RIZKY, Z. P.; YOLLA, P. B.; RAMDHAN, D. H.. Particulate matter $2.5\left(\mathrm{PM}_{2.5}\right)$ personal exposure evaluation on mechanics and administrative officers at the motor vehicle testing center at Pulo Gadung, DKI Jakarta. Reviews on Environmental Health, Berlin, v.31, n.1, p.185-186, 2016. DOI: http://doi.org/10.1515/reveh-2015-0056

RODRIGUES, P. C. O.; PINHEIRO, S. L.; JUNGER, W.; IGNOTTI, E.; HACON, S. S.. Variabilidade climática aumenta a morbimortalidade associada ao material particulado. Revista de Saúde Pública, São Paulo, v.51, n.91, p.1-9, 2017. DOI: http://doi.org/10.11606/s1518-8787.2017051006952

SEINFELD, J. H.; PANDIS, S. N.. Atmospheric chemistry and physics: From air pollution to climate change. 3 ed. New York: John Wiley \& Sons, 2006.

VARDAR, Ç. E.; BASARAM, E.; CANSARAN-DUMAN, D.; ARAS, S.. Air-quality biomonitoring: assessment of genotoxicity of air pollution in the Province of Kayseri (Central Anatolia) by use of the lichen Pseudevernia furfuracea (L.) Zopf and amplified fragment-length polymorphism markers. Mutation Research, Amsterdam, v.759, p.43-50, 2014. DOI: http://doi.org/10.1016/j.mrgentox.2013.09.011

WHO. World Health Organization. Air quality guidelines for particulate matter, ozone, nitrogen dioxide and sulfur dioxide. Switzerland: WHO, 2006.

WHO. World Health Organization. Global urban ambient air pollution database. Country grouping, 2016.

WHO. World Health Organization. Burden of disease from household air pollution for 2016. Switzerland: WHO, 2018

A CBPC - Companhia Brasileira de Produção Científica (CNPJ: 11.221.422/0001-03) detém os direitos materiais desta publicação. Os direitos referem-se à publicação do trabalho em qualquer parte do mundo, incluindo os direitos às renovações, expansões e disseminações da contribuição, bem como outros direitos subsidiários. Todos os trabalhos publicados eletronicamente poderão posteriormente ser publicados em coletâneas impressas sob coordenação da Sustenere Publishing, da Companhia Brasileira de Produção Científica e seus parceiros autorizados. Os (as) autores (as) preservam os direitos autorais, mas não têm permissão para a publicação da contribuição em outro meio, impresso ou digital, em português ou em tradução. 